\title{
Comparison of glycyrrhizin content in 25 major kinds of Kampo extracts containing Glycyrrhizae Radix used clinically in Japan
}

\author{
Mitsuhiko Nose ${ }^{1}$ (1) $\cdot$ Momoka Tada $^{1} \cdot$ Rika Kojima $^{1} \cdot K_{\text {Kumiko Nagata }}{ }^{\text {}}$ \\ Shinsuke Hisaka $^{1}$ - Sayaka Masada ${ }^{2}$ - Masato Homma ${ }^{3}$. Takashi Hakamatsuka ${ }^{2}$
}

Received: 27 April 2017 / Accepted: 2 June 2017/Published online: 12 June 2017

(C) The Author(s) 2017

\begin{abstract}
Glycyrrhizae Radix is the most frequently used crude drug in Japan and is prescribed in Kampo medicine for the treatment of a wide range of diseases. The major active ingredient of Glycyrrhizae Radix, glycyrrhizin (GL), has been shown to possess various pharmacological actions, but is also known to cause adverse effects such as pseudoaldosteronism. To avoid the adverse effects of GL, precautions have been indicated on the package inserts of Glycyrrhizae Radix-containing formulas depending on the amount of Glycyrrhizae Radix they contain. However, it remains unknown whether the extraction efficiency of GL from Glycyrrhizae Radix is constant throughout the different combinations of crude drugs in Glycyrrhizae Radixcontaining formulas. To confirm the basis of the safety regulation, in this study we comprehensively determined the GL content of 25 major kinds of Kampo extracts compounding Glycyrrhizae Radix. We found that the GL content per daily dosage in all Kampo extracts are generally proportional to the compounding amount of Glycyrrhizae Radix, except in the case of shoseiryuto (Sho-
\end{abstract}

Electronic supplementary material The online version of this article (doi:10.1007/s11418-017-1101-x) contains supplementary material, which is available to authorized users.

Mitsuhiko Nose

nose@meijo-u.ac.jp

1 Department of Pharmacognosy, Faculty of Pharmacy, Meijo University, 150 Yagotoyama, Tempaku-ku, Nagoya, Aichi 468-8503, Japan

2 Division of Pharmacognosy, Phytochemistry and Narcotics, National Institute of Health Sciences, 1-18-1 Kamiyouga, Setagaya-ku, Tokyo 158-8501, Japan

3 Department of Pharmaceutical Sciences, Faculty of Medicine, University of Tsukuba, 1-1-1 Tenno-dai, Tsukuba, Ibaraki 305-8575, Japan
seiryu-To). We also found that Schisandrae Fructus in Shoseiryu-To decoction caused a lowered $\mathrm{pH}$ condition and drastically decreased the extraction efficacy of GL from Glycyrrhizae Radix. Moreover, we were able to confirm that the extraction efficiency of GL from Glycyrrhizae Radix is dependent on the $\mathrm{pH}$ value of the extraction solvent. The extraction efficiency of GL in the 25 kinds of Kampo extracts was not constant but it correlates significantly with the $\mathrm{pH}$ value of the decoction. Furthermore, the GL contents are well correlated with pseudoaldosteronism incidence data obtained from the Japanese Adverse Drug Event Report (JADER) database on the 25 kinds of Kampo extracts. This suggests that the GL content is a better index to consider to avoid the adverse effects of Glycyrrhizae Radix-containing Kampo formulas.

Keywords Glycyrrhizae Radix · Glycyrrhizin (GL) . Kampo extracts · HPLC $\cdot \mathrm{pH} \cdot$ Pseudoaldosteronism

\section{Introduction}

Glycyrrhizae Radix is the most frequently used crude drug in Japan and is defined in the Japanese Pharmacopoeia as the root and stolon of Glycyrrhiza uralensis Fischer or Glycyrrhiza glabra Linne. The root of Glycyrrhiza plants has also been used as natural food additives for a long time [1]. Glycyrrhizae Radix is prescribed as an active component in multi-drug formulations of Kampo medicine to treat a variety of diseases.

Glycyrrhizin (GL), a major ingredient of Glycyrrhizae Radix, is a triterpenoid saponin [2] and shows various pharmacological actions such as anti-inflammatory [3-5] and anti-allergy effects [6-8]. Purified GL is also used as a medicine for the treatment of chronic hepatitis in Japan 
[9-11], as well as being used as a sweetener of natural origin [12].

Several investigators have reported that excessive and/or long-term administration of Glycyrrhizae Radix-containing Kampo medicines and crude drug products or GL alone frequently leads to pseudoaldosteronism [13-15], such as peripheral edema [16-18], hypokalemia [19, 20] and hypertension $[21,22]$. These adverse effects are thought to result from glycyrrhetinic acid (GA), a major metabolite of GL that inhibits type $211 \beta$-hydroxysteroid dehydrogenase, leading to an increase in cortisol level instead of cortisone in the kidney. The elevated cortisol stimulates the mineralocorticoid receptor resulting in increased sodium retention and potassium excretion [23, 24]. In order to avoid these adverse effects, the amount of Glycyrrhizae Radix in Kampo prescriptions is seen as an important factor. Different precautions are described on ethical and OTC drug package inserts depending on the amount of Glycyrrhizae Radix they contain, e.g., more than or less than $2.5 \mathrm{~g}$ of Glycyrrhizae Radix in compounding Kampo extract formulation. However, it is still unstated whether the same amount of GL in each Kampo prescription is based on the amount of Glycyrrhizae Radix or not.

Therefore, this study aims to determine how the GL content varies in major Kampo prescriptions, and if the extraction efficiency of GL is affected by other constituents and/or crude drugs in compounding Kampo prescriptions.

In the present study, we chose 25 kinds of Kampo formulas containing Glycyrrhizae Radix from the top 20 list of ethical Kampo formulations and the top 30 list of OTC Kampo formulations in Japan (2011). The Kampo extracts were then prepared and their GL contents were determined by high-performance liquid chromatography (HPLC). Moreover, we calculated and compared the extraction efficacy of each Kampo extract. We also evaluated the relationship between the compounding amounts of Glycyrrhizae Radix or the GL content of the 25 kinds of Kampo extracts and the number of reported cases obtained from the Japanese Adverse Drug Event Report (JADER) database to provide recommendations on how to avoid the adverse effects such as pseudoaldosteronism in Kampo formulas compounding Glycyrrhizae Radix.

\section{Materials and methods}

\section{Materials}

Crude drugs for Kampo prescription were purchased from Tsumura \& Co. (Ibaraki, Japan), Tochimoto Tenkaido Co., Ltd. (Osaka, Japan) and Daiko Shoyaku Ltd. (Aichi, Japan). All crude drugs used in this study were Japanese Pharmacopoeia 16th edition (JP XVI) grade. Most of them were crude drugs for preparation of Kampo formulations, and Chrysanthemi Flos and Kasseki were distributed for food and in-pharmacy formulation, respectively. The same lot listed in Table 1 was used throughout all experiments. HPLC-grade acetonitrile and other solvents and chemicals were purchased from Wako (Osaka). A Millipore syringedriven filter unit (Millex-HP, $0.45 \mu \mathrm{m}$ pore size) was purchased from Merck Millipore, Ltd. (Darmstadt, Germany). The standard GL (purity $>94 \%$, HPLC) was kindly provided by Dr. Yukio Ogihara, emeritus professor at Nagoya City University.

\section{Preparation of Kampo extracts compounding Glycyrrhizae Radix and measurement of the $\mathrm{pH}$ values of the decoctions}

A daily dosage of crude drugs compounded according to each Kampo formulas was decocted with $600 \mathrm{ml}$ ion-exchanged and distilled water using an electric heater (HMJ$1000 \mathrm{~N}$; HARIO Co., Ltd., Tokyo, Japan) for $60 \mathrm{~min}$. The decoction was filtered then cooled down to room temperature. The $\mathrm{pH}$ value was measured by a $\mathrm{pH}$ meter (SevenEasy pH; Mettler Toledo, Switzerland) using electrodes (InLab Expert Pro). Finally, the filtrate was lyophilized to powder. The extract was stored at $-20^{\circ} \mathrm{C}$ before use. In the case of Shoseiryuto (Sho-seiryu-To), the extracts of crude drugs and Kampo formulas without one crude drug were prepared in the same manner.

\section{HPLC analysis of GL content in 25 kinds of Kampo extracts and crude drugs}

All procedures were based on JPX VI. Briefly, $50 \mathrm{mg}$ of each powdered Kampo extract was accurately weighed and dissolved with water up to a volume of $50 \mathrm{ml}$. The mixture was filtered with Millex-HP and then subjected to HPLC analysis. The Shimadzu LC-10Avp HPLC series with UVVIS detector and C-R8A (Kyoto, Japan) were used for data acquisition and integration. Separations were carried out in an Inertsil-ODS3 (5 $\mu \mathrm{m}, 4.6 \mathrm{~mm}$ I.D. $\times 150 \mathrm{~mm}, \mathrm{GL}$ Science, Tokyo) with $2 \%$ acetic acid-acetonitrile (60:40) as eluent. The detection wavelength was $254 \mathrm{~nm}$, the flow rate was $1.0 \mathrm{ml} / \mathrm{min}$, and column temperature was $40{ }^{\circ} \mathrm{C}$. The determination of GL content was carried out by absolute calibration curve method.

\section{Effect of pH on the extraction efficiency of GL from Glycyrrhizae Radix}

With reference to JP XVI, powdered crude drugs were accurately weighed and extracted with $100 \mathrm{mM}$ citric buffer ( $\mathrm{pH} 3.5,4.0), 100 \mathrm{mM}$ acetate buffer $(\mathrm{pH} 4.5)$ or $100 \mathrm{mM}$ phosphate buffer $(\mathrm{pH} 2.1,3.0,5.0,6.0,6.8,8.0)$ 
Table 1 List of crude drugs used in the study

\begin{tabular}{|c|c|c|}
\hline Name of the crude drug & Lot no. & Manufacturers \\
\hline Glycyrrhizae Radix & C10891 & Tsumura \& Co. \\
\hline Angelicae Radix & F26171 & Tsumura \& Co. \\
\hline Asiasari Radix & 25039481 & Tsumura \& Co. \\
\hline Astragali Radix & 24008061 & Tsumura \& Co. \\
\hline Bupleuri Radix & F18911 & Tsumura \& Co. \\
\hline Ginseng Radix & F3297 & Tsumura \& Co. \\
\hline Paeoniae Radix & D49651 & Tsumura \& Co. \\
\hline Platycodi Radix & AD2681 & Tsumura \& Co. \\
\hline Polygalae Radix & AE4891 & Tsumura \& Co. \\
\hline Puerariae Radix & H42281 & Tsumura \& Co. \\
\hline Rehmanniae Radix & F00661 & Tsumura \& Co. \\
\hline Saposhnikoviae Radix & AE2041 & Tsumura \& Co. \\
\hline Saussreae Raidx & $3 \mathrm{~F} 10 \mathrm{M}$ & Daiko Shoyaku Ltd. \\
\hline Scutellariae Radix & AJ3161 & Tsumura \& Co. \\
\hline Alismatis Tuber & AA8921 & Tsumura \& Co. \\
\hline Ophiopogonis Tuber & F33241 & Tsumura \& Co. \\
\hline Pinelliae Tuber & DO8801 & Tsumura \& Co. \\
\hline Atractylodis Rhizoma & F29971 & Tsumura \& Co. \\
\hline Cimicifugae Rhizoma & AA9131 & Tsumura \& Co. \\
\hline Cnidii Rhizoma & 23038151 & Tsumura \& Co. \\
\hline Copitidis Rhizoma & AD1381 & Tsumura \& Co. \\
\hline Rhei Rhizoma & AJ1911 & Tsumura \& Co. \\
\hline Sinomeni Caulis et Rhizoma & F47171 & Tsumura \& Co. \\
\hline Zingiberis Processum Rhizoma & AD9651 & Tsumura \& Co. \\
\hline Zingiberis Rhizoma & AE5571 & Tsumura \& Co. \\
\hline Cinnamomi Cortex & D43061 & Tsumura \& Co. \\
\hline Lycii Cortex & 022012003 & Tochimoto Tenkaido Co. Ltd. \\
\hline Magnoliae Cortex & AD3571 & Tsumura \& Co. \\
\hline Moutan Cortex & AJ9201 & Tsumura \& Co. \\
\hline Uncariae Uncis cum Ramulus & AE4331 & Tsumura \& Co. \\
\hline Citri Unshu Pericarpium & AE2911 & Tsumura \& Co. \\
\hline Euodiae Fructus & 25036841 & Tsumura \& Co. \\
\hline Forsythiae Fructus & AD2891 & Tsumura \& Co. \\
\hline Gardeniae Fructus & AJ8671 & Tsumura \& Co. \\
\hline Oryzae Fructus & 039007005 & Tochimoto Tenkaido Co. Ltd. \\
\hline Schisandrae Fructus & $\mathrm{AJ} 2741$ & Tsumura \& Co. \\
\hline Zizyphi Fructus & D35531 & Tsumura \& Co. \\
\hline Armeniacae Semen & AA8781 & Tsumura \& Co. \\
\hline Longan Arillus & 022813003 & Tochimoto Tenkaido Co. Ltd. \\
\hline Nelumbis Semen & F24001 & Tsumura \& Co. \\
\hline Plantaginis Semen & $1 \mathrm{I} 05 \mathrm{M}$ & Daiko Shoyaku Ltd. \\
\hline Zizyphi Semen & AE0271 & Tsumura \& Co. \\
\hline Ephedra Herba & F17391 & Tsumura \& Co. \\
\hline Menthae Herba & AA890 & Tsumura \& Co. \\
\hline Perillae Herba & AJ0511 & Tsumura \& Co. \\
\hline Chrysanthemi Flos & 0L13 & Daiko Shoyaku Ltd. \\
\hline Schizonepetae Spica & AD6531 & Tsumura \& Co. \\
\hline Polyporus & AD0201 & Tsumura \& Co. \\
\hline Poria & F19561 & Tsumura \& Co. \\
\hline Asini Corii Collas & 3F03M & Daiko Shoyaku Ltd. \\
\hline
\end{tabular}


Table 1 continued

\begin{tabular}{lll}
\hline Name of the crude drug & Lot no. & Manufacturers \\
\hline Ostreae Testa & AE2701 & Tsumura \& Co. \\
Fossilia Ossis Mastodi & AJ7681 & Tsumura \& Co. \\
Gypsum Fibrosum & C52151 & Tsumura \& Co. \\
Kasseki & OK02 & Daiko Shoyaku Ltd. \\
Natrium Sulfuricum & F00615 & Tomita Pharmaceutical Co. Ltd.
\end{tabular}

instead of diluted ethanol. Then, the GL content was determined by HPLC.

\section{Statistical analysis}

The correlation analyses (i) between the extraction efficiency and the $\mathrm{pH}$ value of the decoction, and (ii) the compounding amounts of Glycyrrhizae Radix or the GL contents and the number of reported cases concerning pseudoaldosteronism, were performed using Pearson's correlation.

\section{Results and discussion}

\section{GL content in crude drugs and Glycyrrhizae Radix as a material}

First, the GL content in Glycyrrhizae Radix used in this study was determined by HPLC, based on the quantitative method described in JP XVI. It was found that the lot used in this study contained $54.5 \pm 2.5 \mathrm{mg}$ of GL per $1 \mathrm{~g}$ of dried Glycyrrhizae Radix.

It has generally been believed that a higher specific surface area meant higher extraction efficiency in preparing plant extracts to obtain the target compounds using appropriate solvent for a particular material, such as crude drugs. Thus, considering this principle we compared the extraction efficiency of GL in decoction using cut crude drug and powdered crude drug of Glycyrrhizae Radix. Each $2 \mathrm{~g}$ of cut crude drug or powdered crude drug prepared from the same lot of Glycyrrhizae Radix was decocted with $600 \mathrm{ml}$ of water for $60 \mathrm{~min}$ and the extract was then lyophilized to powder. The yield of the extract and GL content were calculated and compared. We observed that the weight of the extracts was almost the same (average $0.80 \mathrm{~g}$ from cut crude drug and average $0.74 \mathrm{~g}$ from powdered crude drug) and the GL content was also almost equal, as shown in Fig. 1. These results also revealed that GL can be quantitatively extractable in this condition. The reason why the extraction efficiency was not affected by the size and granularity of the crude drug could be due to the higher water solubility of GL. In any case, we

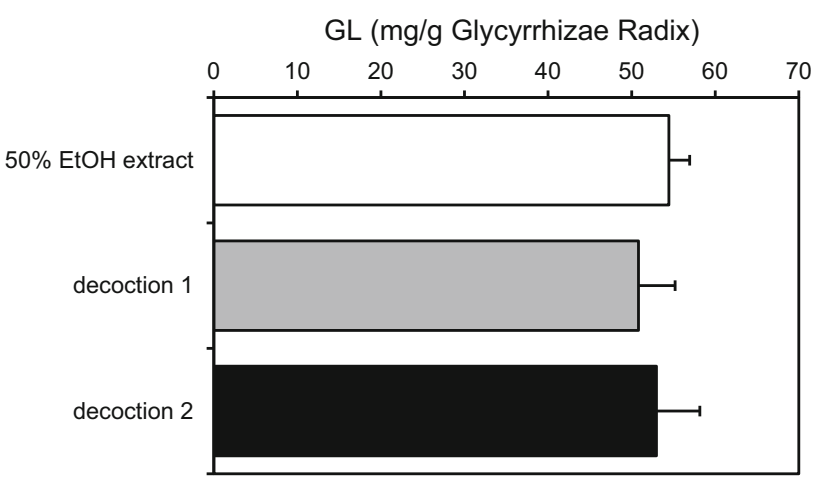

Fig. 1 Determination of glycyrrhizin (GL) content in Glycyrrhizae Radix used in this study. The crude drug (Lot No. C10891, Tsumura \& Co.) was extracted with $50 \%$ ethanol solution as described in JPX VI and the GL content was determined by high-performance liquid chromatography. Decoction 1 was prepared with cut crude drug and decoction 2 was prepared with powdered crude drug. Each column represents the mean \pm SEM of three samples

have shown that we do not have to be apprehensive about the difference in extraction efficiency of GL with regard to the size and granularity of the crude drug, Glycyrrhizae Radix, when we prepare and determine the GL content in each Kampo medicine compounding Glycyrrhizae Radix.

\section{GL content in 25 kinds of Kampo extracts compounding Glycyrrhizae Radix}

We chose 25 kinds of Kampo formulas from the top 20 ethical Kampo formulations and top 30 OTC Kampo formulations in Japan (2011) for the determination of GL content in this study (Table 2). In particular, shakuyakukanzoto (Shakuyakukanzo-To) shoseiryuto (Sho-seiryu-To) and hangesyashinto (Hange-shashin-To) were selected as formulations compounding 4, 3.0, and $2.5 \mathrm{~g}$ of Glycyrrhizae Radix. In addition, gorinsan (Gorin-San), unkeito (Unkei-To), bakumondoto (Bakumondo-To), boiogito (Boi-ogi-To), bofutsushosan (Bofu-tsusho-San), saireito (Sairei-To), shosaikoto (Shosaiko-To), saibokuto (Saiboku-To), kakkonto (Kakkon-To) and keishikaryukotsuboreito (Keishi-ka-ryukotsu-borei-To) were selected as formulations compounding $2.0 \mathrm{~g}$ of Glycyrrhizae Radix, while maoto (Mao-To), saikokeishito (Saiko-keishi-To), seishinrenshiin (Seishin-renshi-In), 
Table 2 List of Kampo prescriptions used in the study

\begin{tabular}{|c|c|}
\hline \multicolumn{2}{|c|}{ shakuyakukanzoto (Shakuyaku-kanzo-To) } \\
\hline Paeoniae Radix & 4.0 \\
\hline Glycyrrhizae Radix & 4.0 \\
\hline \multicolumn{2}{|l|}{ shoseiryuto (Sho-seiryu-To) } \\
\hline Ephedra Herba & 3.0 \\
\hline Paeoniae Radix & 3.0 \\
\hline Zingiberis Processum Rhizoma & 3.0 \\
\hline Glycyrrhizae Radix & 3.0 \\
\hline Cinnamomi Cortex & 3.0 \\
\hline Asiasari Radix & 3.0 \\
\hline Schisandrae Fructus & 3.0 \\
\hline Pinelliae Tuber & 6.0 \\
\hline \multicolumn{2}{|l|}{ hangeshashinto (Hange-shashin-To) } \\
\hline Pinelliae Tuber & 5.0 \\
\hline Scutellariae Radix & 2.5 \\
\hline Zingiberis Processum Rhizoma & 2.5 \\
\hline Ginseng Radix & 2.5 \\
\hline Glycyrrhizae Radix & 2.5 \\
\hline Zizyphi Fructus & 2.5 \\
\hline Copitidis Rhizoma & 1.0 \\
\hline \multicolumn{2}{|l|}{ gorinsan (Gorin-San) } \\
\hline Poria & 5.0 \\
\hline Angelicae Radix & 3.0 \\
\hline Scutellariae Radix & 3.0 \\
\hline Glycyrrhizae Radix & 2.0 \\
\hline Paeoniae Radix & 2.0 \\
\hline Gardeniae Fructus & 2.0 \\
\hline \multicolumn{2}{|l|}{ unkeito (Unkei-To) } \\
\hline Pinelliae Tuber & 5.0 \\
\hline Ophiopogonis Tuber & 10.0 \\
\hline Angelicae Radix & 2.0 \\
\hline Cnidii Rhizoma & 2.0 \\
\hline Paeoniae Radix & 2.0 \\
\hline Ginseng Radix & 2.0 \\
\hline Cinnamomi Cortex & 2.0 \\
\hline Moutan Cortex & 2.0 \\
\hline Glycyrrhizae Radix & 2.0 \\
\hline Zingiberis Rhizoma & 0.3 \\
\hline Euodiae Fructus & 3.0 \\
\hline Asini Corii Collas & 2.0 \\
\hline \multicolumn{2}{|l|}{ bakumondoto (Bakumondo-To) } \\
\hline Ophiopogonis Tuber & 10.0 \\
\hline Pinelliae Tuber & 5.0 \\
\hline Zizyphi Fructus & 3.0 \\
\hline Ginseng Radix & 2.0 \\
\hline Glycyrrhizae Radix & 2.0 \\
\hline Oryzae Fructus & 5.0 \\
\hline \multicolumn{2}{|l|}{ boiogito (Boi-ogi-To) } \\
\hline Sinomeni Caulis et Rhizoma & 4.0 \\
\hline Astragali Radix & 5.0 \\
\hline
\end{tabular}

Table 2 continued

Atractylodis Rhizoma 3.0

Zingiberis Rhizoma $\quad 1.0$

Zizyphi Fructus $\quad 4.0$

Glycyrrhizae Radix $\quad 2.0$

bofutsushosan (Bofu-tsusho-San)

Angelicae Radix $\quad 1.2$

Paeoniae Radix $\quad 1.2$

Cnidii Rhizoma $\quad 1.2$

Gardeniae Fructus $\quad 1.2$

Forsythiae Fructus $\quad 1.2$

Menthae Herba $\quad 1.2$

Zingiberis Rhizoma $\quad 0.4$

Schizonepetae Spica $\quad 1.2$

Saposhnikoviae Radix $\quad 1.2$

Ephedra Herba $\quad 1.2$

Natrium Sulfuricum $\quad 0.6$

Atractylodis Rhizoma $\quad 2.0$

Platycodi Radix $\quad 2.0$

Scutellariae Radix $\quad 2.0$

Glycyrrhizae Radix $\quad 2.0$

Gypsum Fibrosum $\quad 2.0$

Kasseki 3.0

saireito (Sai-rei-To)

Bupleuri Radix $\quad 5.0$

Pinelliae Tuber $\quad 4.0$

Zingiberis Rhizoma $\quad 1.0$

Scutellariae Radix $\quad 3.0$

Zizyphi Fructus $\quad 2.5$

Ginseng Radix $\quad 2.5$

Glycyrrhizae Radix $\quad 2.0$

Alismatis Tuber $\quad 5.0$

Polyporus $\quad 3.0$

Poria $\quad 3.0$

Atractylodis Rhizoma $\quad 3.0$

Cinnamomi Cortex $\quad 2.5$

shosaikoto (Sho-saiko-To)

Bupleuri Radix $\quad 6.0$

Pinelliae Tuber $\quad 5.0$

Scutellariae Radix $\quad 3.0$

Ginseng Radix $\quad 3.0$

Zizyphi Fructus $\quad 3.0$

Zingiberis Rhizoma $\quad 1.0$

Glycyrhizae Radix $\quad 2.0$

saibokuto (Sai-boku-To)

Bupleuri Radix $\quad 7.0$

Pinelliae Tuber $\quad 5.0$

Zingiberis Rhizoma $\quad 1.0$

Scutellariae Radix $\quad 3.0$

Zizyphi Fructus $\quad 3.0$

Ginseng Radix $\quad 3.0$ 
Table 2 continued

\begin{tabular}{|c|c|}
\hline Glycyrrhizae Radix & 2.0 \\
\hline Poria & 5.0 \\
\hline Magnoliae Cortex & 3.0 \\
\hline Perillae Herba & 2.0 \\
\hline \multicolumn{2}{|l|}{ kakkonto (Kakkon-To) } \\
\hline Puerariae Radix & 8.0 \\
\hline Ephedra Herba & 4.0 \\
\hline Zingiberis Rhizoma & 1.0 \\
\hline Zizyphi Fructus & 4.0 \\
\hline Cinnamomi Cortex & 3.0 \\
\hline Paeoniae Radix & 3.0 \\
\hline Glycyrrhizae Radix & 2.0 \\
\hline \multicolumn{2}{|c|}{ keishikaryukotsuboreito (Keishi-ka-ryukotsu-borei-To) } \\
\hline Cinnamomi Cortex & 3.0 \\
\hline Paeoniae Radix & 3.0 \\
\hline Zizyphi Fructus & 3.0 \\
\hline Zingiberis Rhizoma & 3.0 \\
\hline Glycyrrhizae Radix & 2.0 \\
\hline Fossilia Ossis Mastodi & 2.0 \\
\hline Ostreae Testa & 3.0 \\
\hline \multicolumn{2}{|l|}{ maoto (Mao-To) } \\
\hline Ephedra Herba & 4.0 \\
\hline Armeniacae Semen & 4.0 \\
\hline Cinnamomi Cortex & 3.0 \\
\hline Glycyrrhizae Radix & 1.5 \\
\hline \multicolumn{2}{|c|}{ saikokeishito (Saiko-keishi-To) } \\
\hline Bupleuri Radix & 5.0 \\
\hline Pinelliae Tuber & 4.0 \\
\hline Cinnamomi Cortex & 2.0 \\
\hline Paeoniae Radix & 2.0 \\
\hline Scutellariae Radix & 2.0 \\
\hline Ginseng Radix & 2.0 \\
\hline Zizyphi Fructus & 2.0 \\
\hline Glycyrhizae Radix & 1.5 \\
\hline Zingiberis Rhizoma & 1.0 \\
\hline \multicolumn{2}{|c|}{ seishinrenshiin (Seishin-renshi-In) } \\
\hline Ophiopogonis Tuber & 4.0 \\
\hline Poria & 4.0 \\
\hline Ginseng Radix & 3.0 \\
\hline Plantaginis Semen & 3.0 \\
\hline Scutellariae Radix & 3.0 \\
\hline Astragali Radix & 2.0 \\
\hline Glycyrhizae Radix & 1.5 \\
\hline Nelumbis Semen & 4.0 \\
\hline Lycii Cortex & 2.0 \\
\hline \multicolumn{2}{|c|}{ yokukansankachinpihange (Yokukan-San-ka-chinpi-hange) } \\
\hline Angelicae Radix & 3.0 \\
\hline Cnidii Rhizoma & 3.0 \\
\hline Poria & 4.0 \\
\hline Atractylodis Rhizoma & 4.0 \\
\hline
\end{tabular}

Table 2 continued

\begin{tabular}{lc}
\hline Bupleuri Radix & 2.0 \\
Pinelliae Tuber & 5.0 \\
Glycyrhizae Radix & 1.5 \\
Citri Unshu Pericarpium & 3.0 \\
Uncariae Uncis cum Ramulus & 3.0 \\
hochuekkito (Hochu-ekki-To) & \\
Ginseng Radix & 4.0 \\
Atractylodis Rhizoma & 4.0 \\
Astragali Radix & 4.0 \\
Angelicae Radix & 3.0 \\
Citri Unshu Pericarpium & 2.0 \\
Zizyphi Fructus & 2.0 \\
Bupleuri Radix & 1.0 \\
Glycyrhizae Radix & 1.5 \\
Zingiberis Rhizoma & 0.5 \\
Cimicifugae Rhizoma & 0.5
\end{tabular}

kamishoyosan (Kami-shoyo-San)

Angelicae Radix

Atractylodis Rhizoma $\quad 3.0$

Bupleuri Radix $\quad 3.0$

Gardeniae Fructus $\quad 2.0$

Zingiberis Rhizoma $\quad 1.0$

Paeoniae Radix $\quad 3.0$

Poria $\quad 3.0$

Moutan Cortex 2.0

Glycyrhizae Radix $\quad 1.5$

Menthae Herba $\quad 1.0$

juzentaihoto (Juzen-taiho-To)

Ginseng Radix $\quad 3.0$

Astragali Radix $\quad 3.0$

Atractylodis Rhizoma $\quad 3.0$

Poria $\quad 3.0$

Angelicae Radix $\quad 3.0$

Paeoniae Radix $\quad 3.0$

Rehmanniae Radix $\quad 3.0$

Cinnamomi Cortex $\quad 3.0$

Glycyrhizae Radix $\quad 1.5$

yokukansan (Yokukan-San)

Angelicae Radix $\quad 3.0$

Cnidii Rhizoma $\quad 3.0$

Poria 4.0

Atractylodis Rhizoma $\quad 4.0$

Bupleuri Radix $\quad 2.0$

Glycyrhizae Radix $\quad 1.5$

Uncariae Uncis cum Ramulus $\quad 3.0$

chotosan (Choto-San)

Uncariae Uncis cum Ramulus $\quad 3.0$

Citri Unshu Pericarpium $\quad 3.0$

Chrysanthemi Flos $\quad 2.0$

Saposhnikoviae Radix $\quad 2.0$ 
Table 2 continued

\begin{tabular}{|c|c|}
\hline Pinelliae Tuber & 3.0 \\
\hline Ophiopogonis Tuber & 3.0 \\
\hline Poria & 3.0 \\
\hline Ginseng Radix & 2.0 \\
\hline Zingiberis Rhizoma & 1.0 \\
\hline Glycyrhizae Radix & 1.0 \\
\hline Gypsum Fibrosum & 5.0 \\
\hline \multicolumn{2}{|l|}{ kamikihito (Kami-kihi-To) } \\
\hline Ginseng Radix & 3.0 \\
\hline Poria & 3.0 \\
\hline Longan Arillus & 3.0 \\
\hline Angelicae Radix & 2.0 \\
\hline Bupleuri Radix & 3.0 \\
\hline Glycyrhizae Radix & 1.0 \\
\hline Zizyphi Fructus & 2.0 \\
\hline Zingiberis Rhizoma & 0.5 \\
\hline Atractylodis Rhizoma & 3.0 \\
\hline Zizyphi Semen & 3.0 \\
\hline Astragali Radix & 3.0 \\
\hline Polygalae Radix & 2.0 \\
\hline Gardeniae Fructus & 2.0 \\
\hline Saussreae Raidx & 1.0 \\
\hline Moutan Cortex & 2.0 \\
\hline \multicolumn{2}{|l|}{ ninjinyoeito (Ninjin-yoei-To) } \\
\hline Ginseng Radix & 3.0 \\
\hline Angelicae Radix & 4.0 \\
\hline Paeoniae Radix & 2.0 \\
\hline Rehmanniae Radix & 4.0 \\
\hline Atractylodis Rhizoma & 4.0 \\
\hline Poria & 4.0 \\
\hline Cinnamomi Cortex & 2.5 \\
\hline Astragali Radix & 1.5 \\
\hline Citri Unshu Pericarpium & 2.0 \\
\hline Polygalae Radix & 2.0 \\
\hline Schisandrae Fructus & 1.0 \\
\hline Glycyrhizae Radix & 1.0 \\
\hline \multicolumn{2}{|l|}{ rikkunshito (Rikkunshi-To) } \\
\hline Ginseng Radix & 4.0 \\
\hline Atractylodis Rhizoma & 4.0 \\
\hline Poria & 4.0 \\
\hline Pinelliae Tuber & 4.0 \\
\hline Citri Unshu Pericarpium & 2.0 \\
\hline Zizyphi Fructus & 2.0 \\
\hline Glycyrhizae Radix & 1.0 \\
\hline Zingiberis Rhizoma & 0.5 \\
\hline
\end{tabular}

yokukansankachinpihange (Yokukan-San-ka-chinpi-hange), hochuekikito (Hochu-ekiki-To), kamishoyosan (Kamishoyo-San), juzentaihoto (Juzen-taiho-To) and yokukansan
(Yokukan-San) were selected as formulations compounding $1.5 \mathrm{~g}$ of Glycyrrhizae Radix. Lastly, chotosan (Choto-San), kamikihito (Kami-kihi-To), ninjinyoeito (Ninjin-yoei-To) and rikkunshito (Rikkunshi-To) were selected as formulations compounding $1.0 \mathrm{~g}$ of Glycyrrhizae Radix.

As shown in Fig. 2 (see also Supplement Table 1), the GL content per daily dosage in each Kampo medicine is generally proportional to the compounding amount of Glycyrrhizae Radix. Figure 3a shows the relationship between the GL content and the compounding amount of Glycyrrhizae Radix. The coefficient of determination among 25 formulas is 0.7752 , and Sho-seiryu-To significantly deviates downward from the correlation curve while Shakuyaku-kanzo-To seems to be highly placed above this correlation curve. When we re-calculate the coefficient of determination among 23 or 24 formulas other than Shoseiryu-To and/or Shakuyaku-kanzo-To, we can see the comparatively good linearity and higher value of the determination coefficient $\left(r^{2}=0.9235\right)$ if we save and except Sho-seiryu-To (Fig. 3b-d). Thus, we can regard Sho-seiryu-To as an aberration to this observation.

\section{Effects of other crude drugs on GL content in Sho-seiryu-To}

In our preliminary experiments, we prepared extracts by combining Glycyrrhizae Radix with other components compounding Sho-seiryu-To such as Pinelliae Tuber, Ephedra Herba, Cinnamomi Cortex, Schisandrae Fructus, Paeoniae Radix, Asiasari Radix, and Zingiberis Processum Rhizoma. Then, the GL content of every combination was determined by HPLC. We observed a significant decrease of GL content when Glycyrrhizae Radix was combined with Schisandrae Fructus containing Sho-seiryu-To (data not shown).

In order to confirm the effect of Schisandrae Fructus on GL content in Sho-seiryu-To, we prepared the extract of Glycyrrhizae Radix alone, the extract of Glycyrrhizae Radix combined with Schisandrae Fructus, and the extract of Shoseiryu-to without Schisandrae Fructus, and determined the GL content. As shown in Fig. 4, the GL content of the extract with Schisandrae Fructus was about half of that in the extract of Glycyrrhizae Radix alone and nearly equal to that in Shoseiryu-To. Furthermore, the GL content increased significantly when Sho-seiryu-To was prepared without Schisandrae Fructus. These results suggest that the low content of GL in Sho-seiryu-To is due to Schisandrae Fructus.

Schisandrae Fructus is the fruit of Schisandra chinensis (Schisandraceae) and contains lignans such as schizandrin and gomisin A. It has been reported that it also contains organic acids such as citric acid, malic acid and tartaric acid [25]. Due to the organic acids, the $\mathrm{pH}$ value could be lowered and the extraction efficiency of GL could be 


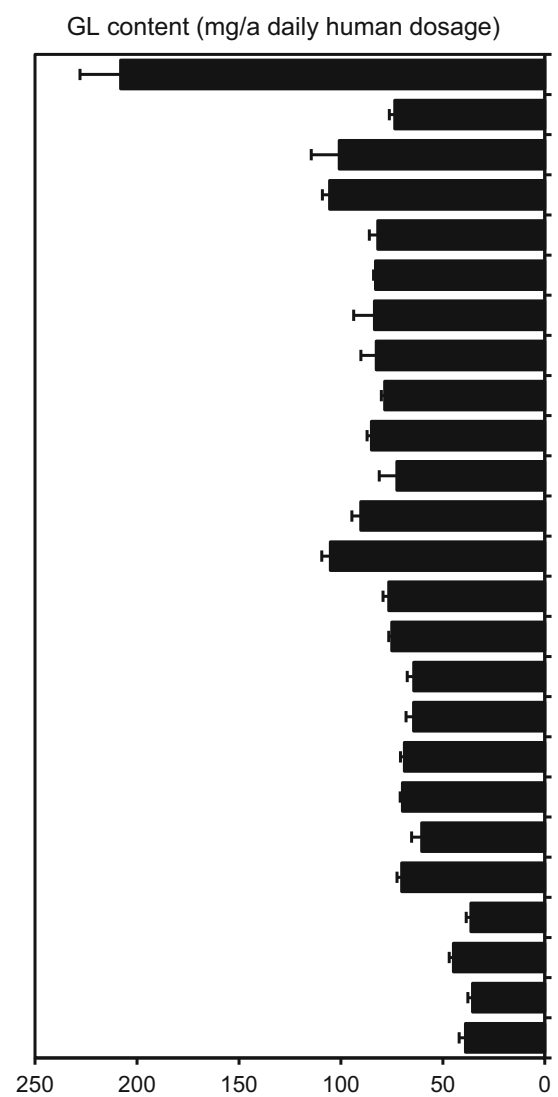

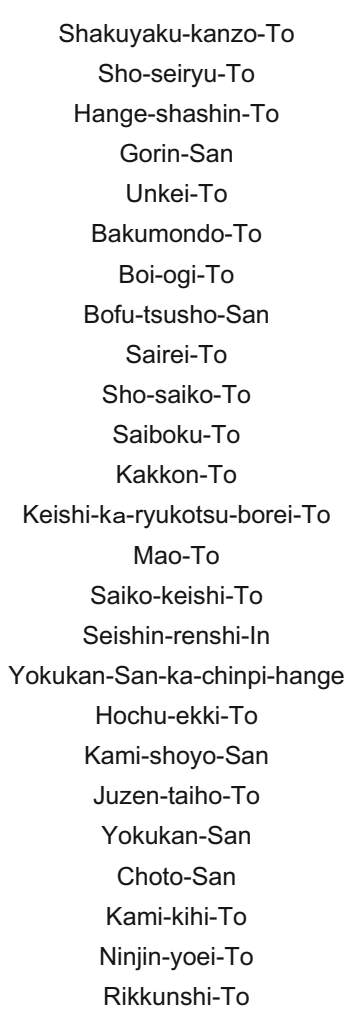

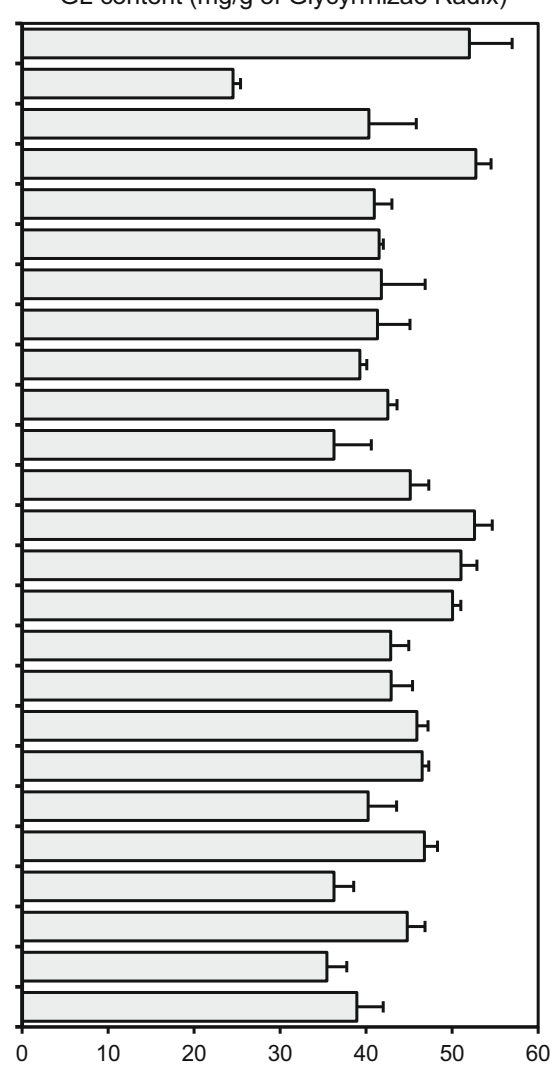

Fig. 2 Determination of glycyrrhizin (GL) content in 25 kinds of Kampo extracts. Each column represents the mean \pm SEM of three samples

decreased. In connection with this, we also measured the $\mathrm{pH}$ value of the decoctions. As shown in Fig. 5, the $\mathrm{pH}$ value in the decoction with Schisandrae Fructus was lower and its $\mathrm{pH}$ was $<3$. The $\mathrm{pH}$ of the decoction of Glycyrrhizae Radix alone was 5.44, the decoction with Schisandrae Fructus was 3.31, and that of the decoction of Sho-seiryu-To was 3.83. The $\mathrm{pH}$ of the decoction of Shoseiryu-to without Schisandrae Fructus increased to 4.98 . These results are comparable to the results of the GL content in each extract; therefore, we think that the $\mathrm{pH}$ in the decoction is a critical factor that could affect the extraction efficiency of GL. Okamura et al. have already reported that low $\mathrm{pH}$ due to the organic acids of Schisandrae Fructus in the decoction caused the inhibition of GL dissolution in Sho-seiryu-To when they demonstrated simultaneous HPLC determination of puerarin, daizein, paeoniflorin, liquiritin, cinnamic acid cinnamaldehyde and GL in 8 kinds of Kampo formulas containing Ephedra Herba [26]. We observed that our findings in the case of Sho-seiryu-To are consistent with their results. On the other hand, Ninjin-yoei-to also contain Schisandrae Fructus but the GL content and the $\mathrm{pH}$ value of the decoction were not affected. We speculate that because the amount of Schisandrae Fructus in Ninjin-yoei-To is smaller resulting in less organic acid, the effect is not as profound as in Sho-
seiryu-To (Fig. 2). Furthermore, we looked for other crude drugs containing organic acids like Schisandrae Fructus and we found Corni Fructus, fruits of Cornus officinalis (Cornaceae). Kampo formulas containing Corni Fructus are Gosha-jinki-Gan, Hachimi-jio-Gan and Rokumi-jio-Gan in ethical and OTC formulations, but fortunately these Kampo prescriptions do not contain Glycyrrhizae Radix. Therefore, the combination between Glycyrrhizae Radix and Corni Fructus was not investigated.

\section{Effect of $\mathrm{pH}$ on the extraction efficiency of GL from Glycyrrhizae Radix}

We clarified the effect of $\mathrm{pH}$ on the extraction efficiency of GL from Glycyrrhizae Radix using powdered crude drug and various buffers.

As shown in Fig. 6, the extraction efficiency showed the sigmoid curve relative to $\mathrm{pH}$. GL was not extracted in the buffer at $\mathrm{pH} 2.1$ and $\mathrm{pH} 3$. It was then gradually dissolved until the extraction efficiency reached maximum at $\mathrm{pH}$ 5.0, and a slight decrease in dissolution was observed with enhanced $\mathrm{pH}$ value. Since the pKa value of GL has been reported as $\mathrm{pKa} 1=3.98, \mathrm{pKa} 2=4.62$ and $\mathrm{pKa} 3=5.17$ [27], the dissolution behavior may be explained by the $\mathrm{pKa}$ value of GL. 

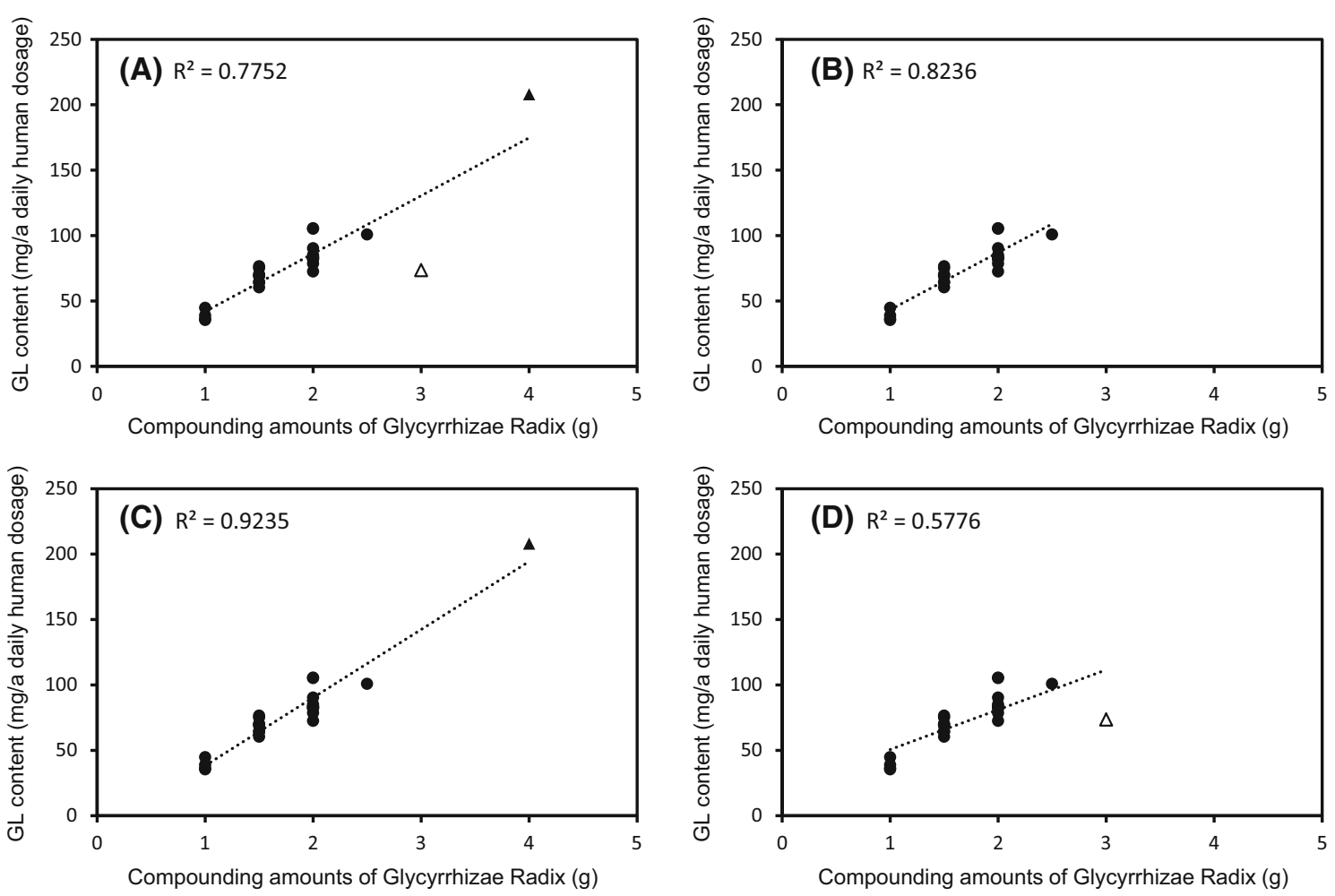

Fig. 3 The relationship between glycyrrhizin (GL) content and amount of Glycyrrhizae Radix compounding 25 kinds of Kampo extracts. a 25 kinds of Kampo extracts, b 23 kinds of Kampo extracts excluding Sho-seiryu-To and Shakuyaku-kanzo-To, c 24 kinds of

Kampo extracts excluding Sho-seiryu-To, d 24 kinds of Kampo extracts excluding Shakuyaku-kanzo-To. Each point represents the mean of three samples. Filled circle 23 kinds of Kampo extracts, open triangle Sho-seiryu-To, filled triangle Shakuyaku-kanzo-To
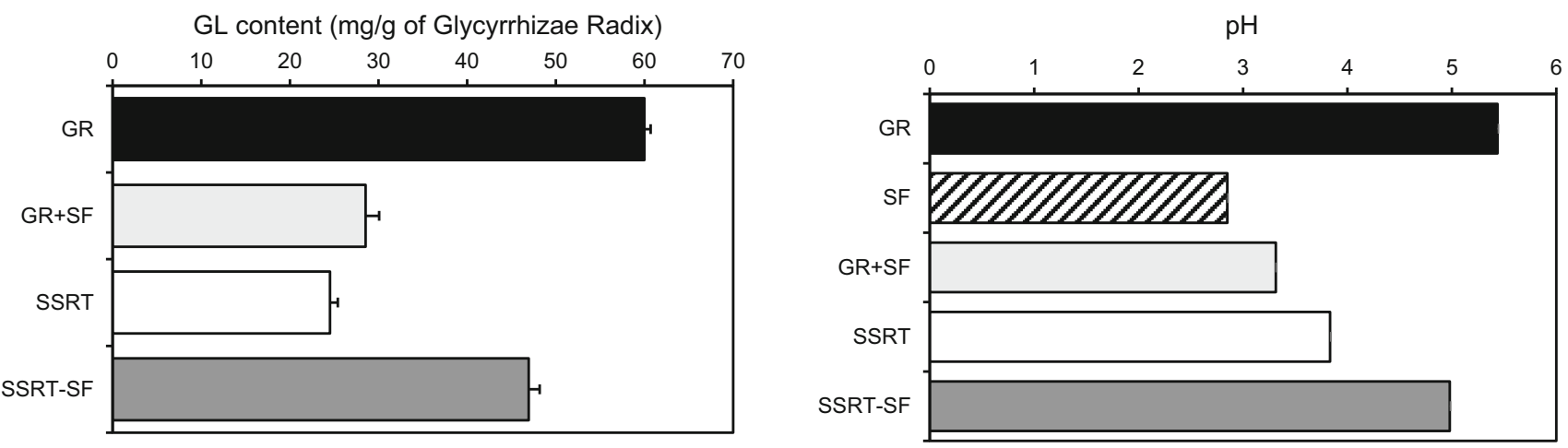

Fig. 4 Effect of Schisandrae Fructus on the extraction efficiency of glycyrrhizin (GL) in Sho-seiryu-To. Each column represents the mean \pm SEM of three samples

In addition, we verified the effect of the $\mathrm{pH}$ value of the solvent on the extraction efficiency of GL from Glycyrrhizae Radix. The powdered Glycyrrhizae Radix was extracted with $100 \mathrm{mM}$ phosphate buffer at $\mathrm{pH} 2.1$ or $\mathrm{pH}$ 6.8. As expected, the GL content in the buffer at $\mathrm{pH} 2.1$ was lower than in the buffer at $\mathrm{pH} 6.8$. Next, the residue extracted with a buffer at $\mathrm{pH} 2.1$ was re-extracted with a buffer at $\mathrm{pH} 6.8$ and eventually GL thought to be contained in the residue was recovered

Fig. 5 Effect of Schisandrae Fructus on the $\mathrm{pH}$ value of the decoction in Sho-seiryu-To. Each column represents the mean \pm SEM of three samples

quantitatively from the buffer with $\mathrm{pH} 6.8$ (Fig. 7). Thus, we have confirmed that the extraction efficiency of GL from its crude drug is dependent on the $\mathrm{pH}$ value of the decoction.

Lastly, we measured the $\mathrm{pH}$ value of the decoction of the 25 kinds of Kampo extracts used in the study and the data were added to the top of the graph in Fig. 6 (Fig. 8). Interestingly, Keishi-ka-ryukotsu-borei-To showed a relatively high $\mathrm{pH}$ value ( $\mathrm{pH}$ 6.3) and its mean GL content was 


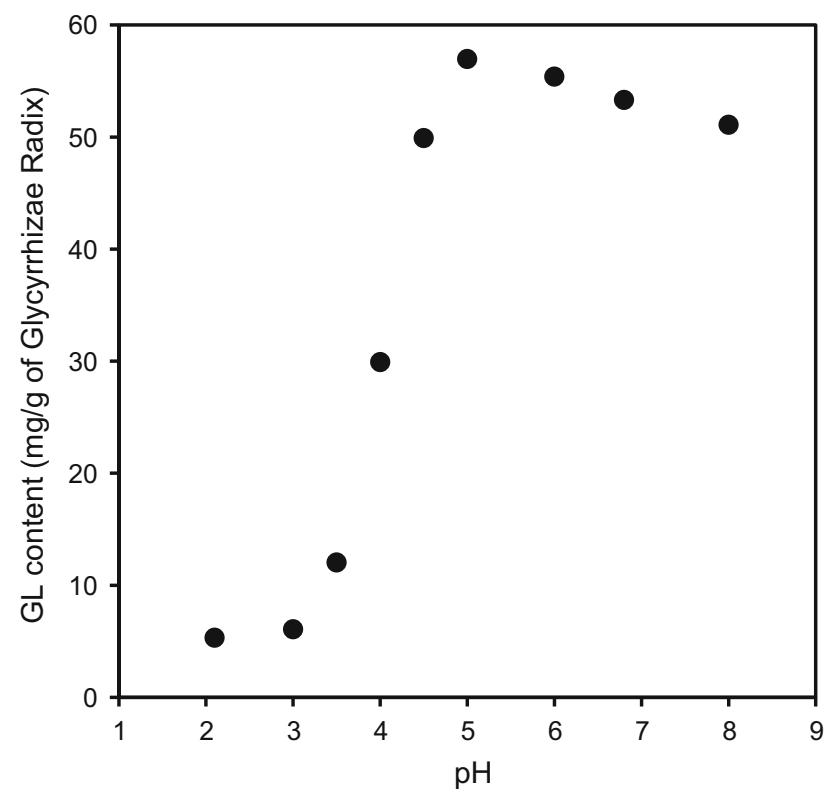

Fig. 6 The relationship between the extraction efficiency of glycyrrhizin (GL) and the $\mathrm{pH}$ value of buffer. Each point represents the mean of three samples

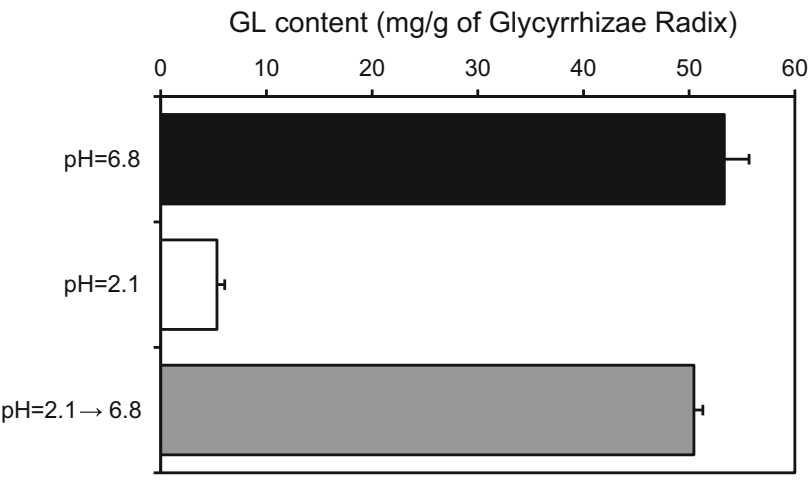

Fig. 7 Effect of pH on the extraction efficiency of glycyrrhizin (GL) from Glycyrrhizae Radix: recovery of the extraction efficiency of GL extracted with $100 \mathrm{mM}$ phosphate buffer $(\mathrm{pH}=6.8)$ from the residue pre-extracted with $100 \mathrm{mM}$ phosphate buffer $(\mathrm{pH}=2.1)$. Each column represents the mean of three samples

$52.6 \mathrm{mg} / \mathrm{g}$ of the crude drug. Keishi-ka-ryukotsu-borei-To contains Fossilia Ossis Mastodi and Ostea Testa, and it has been reported that the $\mathrm{pH}$ value can be around the neutral range because of the calcium carbonate present in the mentioned crude drugs [28]. The correlation analysis showed that the extraction efficiency correlates significantly with the $\mathrm{pH}$ value of the decoction $(r=0.7101$, $p<0.0001)$. These results suggest that the extraction efficiency of GL is basically dependent on the $\mathrm{pH}$ value of the decoction, but other factors such as adsorption to other crude drugs compounding Kampo formulas could also

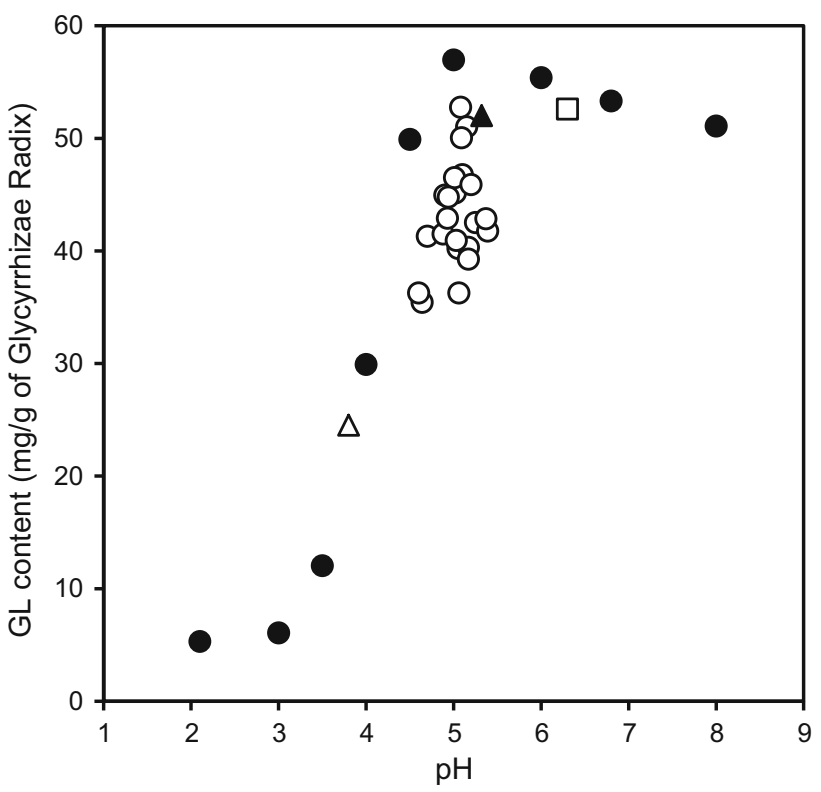

Fig. 8 The relationship between the extraction efficiency of glycyrrhizin (GL) and the pH values of 25 kinds of Kampo extracts. Each point represents the mean of three samples. Open circle buffer, filled circle 22 kinds of Kampo extracts, open triangle Sho-seiryu-To, filled traingle Shakuyaku-kanzo-To, open square Keishi-ka-ryukotsu-boreiTo

decrease the GL content in some Kampo prescriptions [29-32].

The relationship between the GL content and the incidence of adverse effects of Kampo medicine containing Glycyrrhizae Radix

Since the GL content in a daily dosage of Sho-seiryu-To is comparatively lower than the other Kampo prescriptions used in the study, we became interested with the incidence of adverse effects of Sho-seiryu-To. The incidence of adverse effects of Kampo prescriptions used in the study was investigated using the Japanese Adverse Drug Event Report (JADER) database from 2004-2015 by the Pharmaceuticals and Medical Devices Agency (PMDA) in Japan [33]. We chose five preferred terms (pseudoaldosteronism, hypokalemia, hypokalemic syndrome, myopathy and rhabdomyolysis) for typical Glycyrrhizae Radix-induced adverse events in the investigation. As shown in Fig. 9, the most reported cases in Shakuyaku-kanzo-To were due to Glycyrrhizae Radix, and the same tendency was observed with YokukanSan. In the case of Sho-seiryu-To, the low GL content could be the reason for the low incidence of pseudoaldosteronism in addition to the short-term use for nose allergy. On the other hand, Hochu-ekki-To and Juzen-taiho-To which contain a lower amount of GL have numerous reported cases related to 


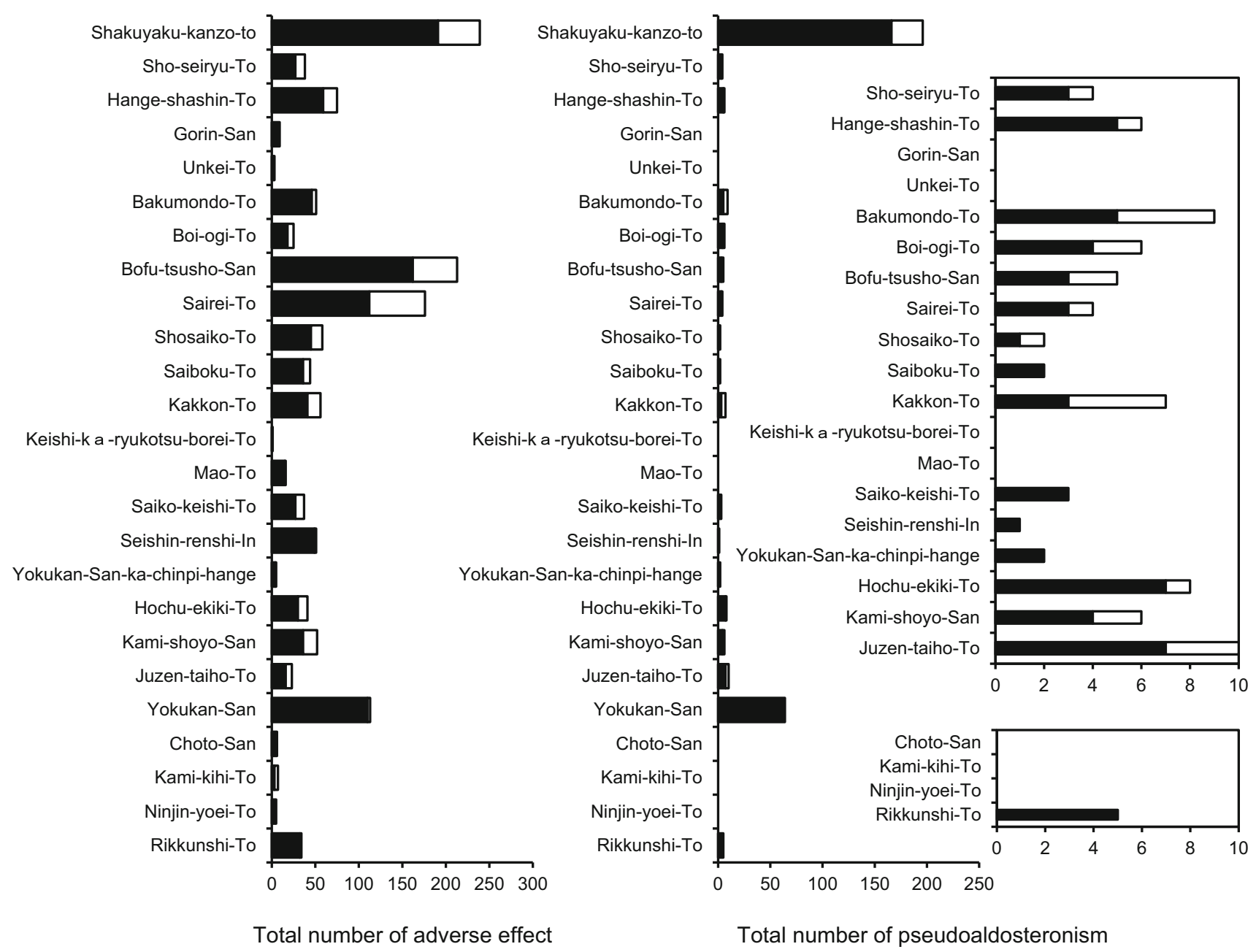

Fig. 9 The incidence of adverse effects of Kampo formulas containing Glycyrrhizae Radix. Closed column represents the incidence from using ethical Kampo extract formulations and opened column represents the incidence from using OTC Kampo extract formulations, respectively

Glycyrrhizae Radix. This observation may be due to their long-term administration for elderly patients. In addition, we evaluated the relationship between the compounding amounts of Glycyrrhizae Radix in 25 kinds of Kampo formulas and the number of reported cases concerning Glycyrrhizae Radix-induced adverse events and we found that the compounding amounts may be associated with pseudoaldosteronism $(r=0.6447, p<0.0005)$. Moreover, we found much better correlation when the GL content was considered instead of the compounding amount of Glycyrrhizae Radix $(r=0.7757, p<0.0001)$. These results suggest that the actual GL content is a better index to consider in order to avoid the adverse effects of Glycyrrhizae Radix-containing formulas. Since there are numerous variations of Glycyrrhizae Radix on the market, it is also possible that there is a certain level of variation in the GL content among the manufacturers of pharmaceuticals even in the same Kampo formula. Thus, further analytical and epidemiological studies are needed.

\section{Conclusion}

In this study, we determined the GL content of the 25 major kinds of Kampo extracts compounding Glycyrrhizae Radix in Japan. We found that the GL content per daily dosage in each Kampo medicine is generally proportional to the compounding amount of Glycyrrhizae Radix. We also clarified that the extraction efficiency of GL in the decoction is not constant and is basically dependent on the $\mathrm{pH}$ value of the decoction. Moreover, the correlation analysis with Glycyrrhizae Radix-induced adverse events obtained from JADER suggested that the actual GL content is a better index to consider in order to avoid the adverse effects of Glycyrrhizae Radix-containing Kampo formulas.

Acknowledgement This study was supported by a Health and Labour Sciences Research Grant for 'Research on Regulatory Science of Pharmaceuticals and Medical Devices' from Ministry of Health, Labour and Welfare. 


\section{Compliance with ethical standards}

Conflict of interest The authors declare that they have no conflict of interest.

Open Access This article is distributed under the terms of the Creative Commons Attribution 4.0 International License (http://crea tivecommons.org/licenses/by/4.0/), which permits use, duplication, adaptation, distribution and reproduction in any medium or format, as long as you give appropriate credit to the original author(s) and the source, provide a link to the Creative Commons license and indicate if changes were made.

\section{References}

1. Notification No. 65 (2011) The Japanese Pharmacopia 16th ed. "Glycyrrhiza", Ministry of Health and Welfare, Japan, pp 1649-1650. http://www.mhlw.go.jp/topics/bukyoku/iyaku/ yakkyoku/ehglish.html. Accessed 1 Dec 2016

2. Beaton JM, Spring FS (1956) Triterpenoids. Part LI. The isolation and characterization of glabric acid, a new triterpenoid acid from liquorice root. J Chem Soc. doi:10.1039/JR9560002417

3. Finney RS, Somers GF (1958) The anti-inflammatory activity of glycyrrhetinic acid and derivatives. J Pharm Pharmacol 10:613-620

4. Shibata S (2000) A drug over the millennia: pharmacognosy, chemistry, and pharmacology of licorice. Yakugaku Zasshi 120:849-862

5. Baltina LA (2003) Chemical modification of glycyrrhizic acid as a route to new bioactive compounds for medicine. Curr Med Chem 10:155-171

6. Raphael TJ, Kuttan G (2003) Effect of naturally occurring triterpenoids glycyrrhizic acid, ursolic acid, oleanolic acid and nomilin on the immune system. Phytomedicine 10:483-489

7. Ram A, Mabalirajan U, Das M, Bhattacharya I, Dinda AK, Gangal SV, Ghosh B (2006) Glycyrrhizin alleviates experimental allergic asthma in mice. Int Immunopharmacol 6:1468-1477

8. Ma C, Ma Z, Liao XL, Liu J, Fu Q, Ma S (2013) Immunoregulatory effects of glycyrrhizic acid exerts anti-asthmatic effects via modulation of Th1/Th2 cytokines and enhancement of CD4(+)CD25(+)Foxp3+ regulatory T cells in ovalbumin-sensitized mice. J Ethnopharmacol 148:755-762

9. Kuroyanagi T, Kurisu A, Sugiyama H, Saito M (1962) Studies on experimental allergic hepatitis. 2. Effect of prednisolone and glycyrrhizin on experimental allergic hepatitis. Jpn J Med Prog 49:458-465

10. Coon JT, Ernst E (2004) Complementary and alternative therapies in the treatment of chronic hepatitis $\mathrm{C}$ : a systematic review. J Hepatol 40:491-500

11. Asl MN, Hosseinzadeh H (2008) Review of pharmacological effects of Glycyrrhiza sp. and its bioactive compounds. Phytother Res 22:709-724

12. Morris JA (1976) Sweetening agents from natural sources. Lloydia 39:25-38

13. Conn JW, Rovner DR, Cohen EL (1968) Licorice-induced pseudoaldosteronism. Hypertension, hypokalemia, aldosteronopenia, and suppressed plasma renin activity. JAMA 205:492-496

14. Terasawa K, Bandoh M, Tosa H, Hirate J (1986) Disposition of glycyrrhetic acid and its glycosides in healthy subjects and patients with pseudoaldosteronism. J Pharmacobiodyn 9:98-100

15. Takeda R, Morimoto S, Uchida $K$, Nakai $T$, Miyamoto $M$, Hashiba T, Yoshimitsu K, Kim KS, Miwa U (1979) Prolonged pseudoaldosteronism induced by glycyrrhizin. Endocrinol Jpn 26:541-547

16. Crampton JH (1961) Glycyrrhizinophilia as a cause of edema. Bull Mason Clin 15:89-92

17. Bernardi M, D'Intino PE, Trevisani F, Cantelli-Forti G, Raggi MA, Turchetto E, Gasbarrini G (1994) Effects of prolonged ingestion of graded doses of licorice by healthy volunteers. Life Sci 55:863-872

18. Johns C (2009) Glycyrrhizic acid toxicity caused by consumption of licorice candy cigars. CJEM 11:94-95

19. Strong JA (1951) Serum potassium deficiency during treatment with sodium PAS and liquorice extract. Br Med J 2:998-1002

20. Kageyama Y, Suzuki H, Saruta T (1992) Glycyrrhizin induces mineralocorticoid activity through alterations in cortisol metabolism in the human kidney. J Endocrinol 135:147-152

21. Girerd RJ, Rassaert CL, Di Pasquale G, Kroc RL (1958) Production of experimental hypertension and cardiovascular renal lesions with licorice and ammoniated glycyrrhizin. Am J Physiol 194:241-245

22. Ploeger B, Mensinga T, Sips A, Seinen W, Meulenbelt J, DeJongh J (2001) The pharmacokinetics of glycyrrhizic acid evaluated by physiologically based pharmacokinetic modeling. Drug Metab Rev 33:125-147

23. Monder C, Stewart PM, Lakshmi V, Valentino R, Burt D, Edwards CR (1989) Licorice inhibits corticosteroid 11 beta-dehydrogenase of rat kidney and liver: in vivo and in vitro studies. Endocrinology 125:1046-1053

24. Tanahashi $\mathrm{T}$, Mune $\mathrm{T}$, Morita $\mathrm{H}$, Tanahashi $\mathrm{H}$, Isomura $\mathrm{Y}$, Suwa T, Daido H, Gomez-Sanchez CE, Yasuda K (2002) Glycyrrhizic acid suppresses type 211 beta-hydroxysteroid dehydrogenase expression in vivo. J Steroid Biochem Mol Biol 80:441-447

25. Lu Y, Chen DF (2009) Analysis of Schisandra chinensis and Schisandra sphenanthera. J Chromotogr 1216:1980-1990

26. Okamura N, Miki H, Orii H, Masaoka Y, Yamashita S, Kobayashi H, Yagi A (1999) Simultaneous high-performance liquid chromatographic determination of puerarin, daidzin, paeoniflorin, liquiritin, cinnamic acid, cinnamaldehyde and glycyrrhizin in Kampo medicines. J Pharm Biomed Anal 19:603-612

27. Zeng CX, Hu Q (2008) Determination of the polyacid dissociation constants of glycyrrizic acid. Indian J Chem 47A:71-74

28. Arichi S, Tani T, Kubo M (1979) Studies on BUPLEURI RADIX and saikosaponin (1) Determination of saikosaponins in decoctions of prescriptions of Chinese traditional medicine. Med J Kinki Univ 4:59-66

29. Noguchi M, Kubo M, Hayashi T, Ono M (1976) Studies on the pharmaceutical quality evaluation of the crude drug preparation used in orient medicine "Kampoo" (I) Precipitation reaction of the components of Coptidis Rhizoma and these of Glycyrrhizae Radix or Rhei Rhizoma in decoction solution. Shoyakugaku Zasshi 32:104-110

30. Noguchi M (1978) Studies on the pharmaceutical quality evaluation of the crude drug preparation used in orient medicine "Kampoo". II. Precipitation reaction of berberine and glycyrrhizin in aqueous solution. Chem Pharm Bull 26:2624-2629

31. Noguchi M, Kubo M, Hayashi T, Ono M (1978) Studies on the pharmaceutical quality evaluation of the crude drug preparation used in orient medicine "Kampoo". III. Precipitation reaction of glycyrrhizin with alkaloids or alkaloidal crude drugs in aqueous solution. Chem Pharm Bull 26:3652-3657

32. Tomimori T, Toshimoto M (1980) Quantitative variation of glycyrrhizin in the decoction of Glycyrrhizae Radix mixed with other crude drugs. Shoyakugaku Zasshi 34:138-144

33. Pharmaceuticals and Medical Devices Agency. Information about the case report that a side effect is doubted. http://www.info. pmda.go.jp/fsearchnew/jsp/menu_fukusa-you_base.jsp. Accessed Feb 2016 (in Japanese) 